\title{
Approximations for Call-Blocking Probabilities in Multirouting Multicasting Multirate Loss Networks
}

\author{
Tibor Cinkler and László Ast \\ High-Speed Networks Laboratory \\ Department of Telecommunications and Telematics \\ Budapest University of Technology and Economics \\ Pázmany Péter sétány 1/D, H-1117 Budapest, Hungary \\ cinkler@ttt-atm.ttt.bme.hu
}

\begin{abstract}
For the end-to-end call blocking probability computation in multi-rate loss networks the so called reduced load approximation under link independence assumption is often used, because it allows derivation of analytical results. However, its accuracy and extendibility to multirouting or multicasting networks (like the B-ISDN) is seldom studied. This paper proposes new analytical methods for approximating the call blocking probabilities for alternative routing and point-to-multipoint connections. The results are derived for different cases and techniques and further generalised for Whitt's summation bound.
\end{abstract}

\section{Introduction}

The exact (or good approximate) and fast calculation of end-to-end blocking probabilities in modern telecommunication networks is important, because it is the primary measure of the grade of service the network provides to its users [1|2 344. In broadband integrated services digital networks (B-ISDN), where calls of different service classes are routed between two or more different points, the traditional methods, elaborated for single-rate circuit switched (telephone) networks are no longer adequate. The most frequently used originator-destination (OD) pair blocking evaluation technique is the reduced load (RL) approximation. It assumes link independence: the links of a multihop route block independently from each other, i.e., the events "link $i$ blocks" and "link $j$ blocks" are independent of each other, even if both links are of the same route. This assumption is luring, because it allows the calculation of route blocking probabilities by means of product forms [56]. The reduced load approximation determines the route blocking probabilities using blocking probabilities of those links which are along that route [7].

The validity of the link independence assumption has been studied by Roberts, Dziong, Pióro, Blaabjerg and others, and a number of valuable results exist [7/89]. The RL approximation has been generalised for the multi-rate case where the traffic sources generate the load at different (bps) rates [7]. Unfortunately, 
very few results consider its generalisation to multiroute cases (where a set of routes is offered to each OD pair). Furthermore, to our knowledge it is not discussed how they can be applied to multicast routing - an important routing method, where each connection can be built up between more than two or three users and is expected to be in wide use in future multiservice networks. The obtained results were published in 10,11.

In Section 2 we present analytical methods for determining link and path blocking probabilities for multi-rate case. Then we generalise these results for tree blocking probabilities in case of multicasting, and for OD-pair blocking probabilities in case of multirouting (Alternative Routing (AR) only).

\section{Blocking Probability Evaluation Methods}

A route $r$ is a subset of the set of those logical links that belong to the same logical network. In principle, it can be taken as an arbitrary subset but here they will be simple paths connecting OD (Origin-Destination) pairs and trees connecting a subset of all nodes. By convention, each route carries only calls of a single traffic class $m$. That is, if several traffic classes are to be carried, they are represented by parallel routes. The incidence of routes, links and traffic types is expressed by the indicator variables

$$
A_{m j r}=\left\{\begin{array}{cc}
1 & \text { when route } r \text { uses link } j \text { and carries traffic type } m \\
0 & \text { otherwise }
\end{array}\right.
$$

$A_{m j r}$ is not to be interpreted as the amount of bandwidth that route $r$ requires on link $j$. For that purpose we use other variables: $d_{m j}$ will denote the amount of bandwidth (capacity) that a call belonging to traffic type $m$ requires on link $j$. By this notation we implicitly assume that all routes that carry traffic of type $m$ require the same amount of bandwidth on link $j$. Since the bandwidth requirement is associated with the traffic type, this is not seen as a restriction. On the other hand, we allow the bandwidth requirement of calls on a given route to vary along the links of the route. In fact, this is needed if the concept of effective or equivalent bandwidth (see e.g., [12]) is adopted and the involved links have different capacities.

Let $\nu_{r}$ be the offered traffic to route $r$. Let $\nu_{(v, p, m)}$ be the aggregated offered traffic of class $m$ to node pair $p$ in virtual network $v$. Then the traffic carried over route $r$ can be expressed as

$$
\lambda_{r}=\nu_{r}\left(1-L_{r}\right)
$$

where $L_{r}$ is the end-to-end route blocking probability for traffic on route $r$. Clearly, this route blocking probability is defined as the probability of the event that at least one link is blocked along the route.

For traditional single-rate networks it is known that alternative routing (AR) offers some advantages compared to either simple load sharing or fixed routing. The model presented here can quite easily be extended to either sequential or 
dynamic alternative routing, at least if the overflow traffic is approximated by Poisson traffic. This approximation is justified in the case where only a minor amount of traffic overflows. Therefore the approximation for Dynamic AR (DAR) will be more accurate in general then for Sequential AR (SAR), since DAR decreases the overflow by dynamically changing the primary route.

The call blocking probabilities can be considered at different levels:

- link blocking,

- route blocking,

- tree blocking, and

- OD pair (end-to-end) blocking

The call blocking probabilities $L_{r}$ can for a loss network operating under fixed routing receiving Bernoulli-Poisson-Pascal traffic, in principle be found since such a network obeys a product form solution [13]. However, the numerical complexity is prohibitively high for networks of reasonable sizes, and in practice approximations need to be applied.

Here we shall apply two related techniques which both give the route blocking probabilities whenever the blocking probabilities on individual links are known. Furthermore, we approximate OD pair blocking probabilities for multirouting techniques based on the blocking probabilities of routes of the considered OD pair.

\section{Link Blockings}

The analytical blocking probability evaluation for routes, trees and OD-pairs are based on evaluation of link blocking probabilities. Here we assume Complete Sharing, where the traffic of different connections belonging to different traffic classes share the common resources. These methods can simply be generalised for case of Complete Partitioning, Trunk Reservation and other resource sharing/partitioning techniques.

The natural choice for evaluating link-blocking probabilities in a multi-rate network would have been to apply the multi-dimensional Erlang formula, which is very accurate. However, increasing the number of different traffic classes the state-space grows exponentially. Therefore, the projection of this multidimensional state-space to a one-dimensional state-space is preferred.

Consider link $j$ of capacity $C_{j}$ in isolation receiving traffic from the routes going through link $j$. With $\nu_{m, j}$ being the total call arrival rate from traffic class $m$ on link $j$, with $d_{m, j}$ being the bandwidth demand of calls from class $m$, if the holding time as before is assumed equal to 1 for all traffic classes, then the occupancy distribution of link $j$ is found from the Kaufman-Roberts recursion formula (also called stochastic Knapsack) which states (see, e.g., [14])

$$
n p_{j, n}=\sum_{m} \nu_{m, j} d_{m, j} p_{j, n-d_{m, j}} \text { and } \sum_{n=0}^{C_{j}} p_{j, n}=1
$$


where $p_{j, n}$ is the probability of $n$ occupied bandwidth units (circuits) on link $j$. The blocking probability for traffic class $m$ can now be found as

$$
B_{m j}=\sum_{n=C_{j}-d_{m}+1}^{C_{j}} p_{j, n}
$$

For optimisation of large networks, this formula is too complex. The complexity originates from two facts. First the underlying Markov process is multidimensional, and there exists no simple relationship between the blocking probabilities of the individual traffic classes enabling a computation of all the blocking probabilities by just computing the blocking of a single traffic class (e.g., the most narrow one). Secondly, it is a recursive formula and this implies that the complexity increases with increasing capacities of the links.

As a somewhat simpler but in many cases still quite accurate formula, the Pascal approximation can be used. Here, the mean and variance of the occupied bandwidth in case of infinite capacity links is found and a negative binomial (Pascal) distribution with same mean and variance is used, as an approximation of the occupancy distribution. By the usual truncation and by applying (4) with $p_{j, n}$ found from the Pascal approximation the individual blocking probabilities can be found. See, e.g., [15] and 16] for further details. Even though the Pascal approximation is simpler it is also burdened with increasing complexity in increasing capacities.

For high capacity networks where both these formulae become too complicated a simple Gaussian approximation as used and explained in [17] can be useful.

Finally, based on the behavior of the blocking probabilities in the limiting regime [18] where offered traffic and link capacities tend to infinity by the same speed, a very simple approximation could be

$$
\left(1-B_{m, j}\right) \approx\left(1-B_{1, j}\right)^{\left(d_{m, j} / d_{1, j}\right)}
$$

i.e., a call requiring a factor of $d$ more bandwidth units than the most narrow call has a blocking probability as if it was set up as $d$ sequential narrow band calls. For the case where the fraction between the biggest and smallest bandwidth demand is not far from 1 this approximation can be applicable. However as this fraction increases the accuracy deteriorates.

\section{Route Blockings}

\subsection{Reduced Load and Link Independence}

The first method to be presented is the classical reduced load and link independence assumption, see, e.g., [18] and [16]. Here blocking of individual links is assumed to take place independently of other link blockings and the load offered to link $j$ consists not of the total load offered but only the traffic not blocked at other links. To be more precise let $B_{m, j}$ denote the blocking probability of 
traffic class $m$ on link $j$, and let $E_{m, j}\left(\rho_{1}, . ., \rho_{M} ; C_{j}\right)$ be the blocking function which gives the blocking probability of traffic class $m$ on link $j$ when the offered class $m$ bandwidth demand on link $j$ is $\rho_{m}$ and link capacity is $C_{j}$. Then

$$
\begin{gathered}
B_{m j}=E_{m j}\left(\rho_{1 j}, . ., \rho_{M j} ; C_{j}\right) \text { for all } m \text { and } j \\
\lambda_{r}=\nu_{r}\left(1-L_{r}\right) \text { where }\left(1-L_{r}\right)=\prod_{m, k}\left(1-B_{m k}\right)^{A_{m k r}} \\
\rho_{m j}=d_{m j} \sum_{r}\left(1-B_{m j}\right)^{-1} \lambda_{r} A_{m j r}=d_{m j} \nu_{m, j} \text { for all } m \text { and } j
\end{gathered}
$$

where $L_{r}$ is the end-to-end blocking probability for traffic on route $r, \lambda_{r}$ is the carried traffic on route $r$ and $\nu_{m, j}$ is the offered class $m$ traffic to link $j$ under the reduced load approximation.

Clearly, this route blocking probability is defined as the probability of the event that at least one link is blocked along the route.

As seen from (7) then the route blocking probability on any route is easily derived when the link blocking probabilities $B_{m k}$ are known, and they are given as a solution to (6), (17) and (8). By Brouwer's fixed point theorem a solution is known to exist but unfortunately, in the multi-rate case multiple solutions cannot in general be ruled out.

\subsection{Whitt's Summation Bound}

The idea to the second method originates from a result valid for single slot circuit switched networks proved by Whitt in [19].

The result of Whitt (Theorem 1 and Corollary 1.2 in [19]) states that in a single rate loss network operating under fixed routing and receiving Poissonian traffic, the exact route blocking probability $L_{r}^{\text {exact }}$ is upper bounded by the sum of the link blocking probabilities on the links $j$ which route $r$ passes, i.e.,

$$
L_{r}^{\text {exact }} \leq \sum_{j \in r} \tilde{B}_{j}
$$

where $\tilde{B}_{j}$ is the (single class) blocking probability on link $j$ under the assumption that all traffic offered to the routes passing through link $j$ is also offered to link $j$, i.e., not blocked at other links, i.e., the traffic offered to link $j$ is $\sum_{r} \nu_{r} A_{j r}$ (the traffic class index $m$ is omitted).

The main advantage of the generalised Whitt's summation bound is that the difficult solution of the fixed point equations can be avoided, and if the network is only moderately loaded the accuracy can still be sufficient. However, the higher the load is, the larger are the inaccuracies.

This method can easily be generalised for multirate networks:

$$
L_{r}^{\text {exact }} \leq \sum_{m, l} \tilde{B}_{m l} A_{m l r}
$$

where $\tilde{B}_{m l}$ stands for the blocking probability of traffic of class $m$ over link $l$ evaluated without any load reduction. 


\section{$5 \quad$ Multicast Tree Blockings}

Multicasting plays increasingly important role in ATM and IP networks [20].

\subsection{Reduced Load and Link Independence}

The classical reduced load approximation under link independence assumption can be generalised for approximating the connection-blocking probabilities of point-to-multipoint connections (multicast trees) in both single- and multi-rate loss networks.

In modern networks point-to-multipoint connections are increasingly often required. Denote that all multipoint-to-multipoint connections can be built up from point-to-multipoint connections. For example for $K$ nodes a multipointto-multipoint connection can be built up as $K$ one-point to $(K-1)$-point connections. It is much more efficient to use point-to-multipoint trees (Steiner-tree problem) where the information is duplicated (or multiplayed) only in the treebranching points instead of using direct point-to-point connections. In this case the total resource requirement can be significantly reduced.

The blocking probability for establishing a point-to-multipoint connection can be approximated in analogous way to that one presented in Section 4 . The point-to-multipoint connection is considered blocked if more then $k$ among $K$ end-nodes can not be connected where $k<K$. This case is called $k$-reliable multicast. When $k=K$ the multicast is called fully reliable. Approximations for connection blocking probabilities will be given, first for fully reliable multicast, then for the $k$-reliable case.

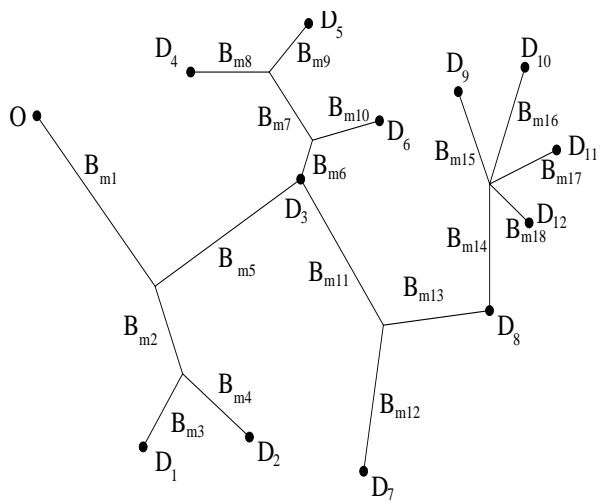

Fig. 1. Dependence of Link and Tree Blocking Probabilities

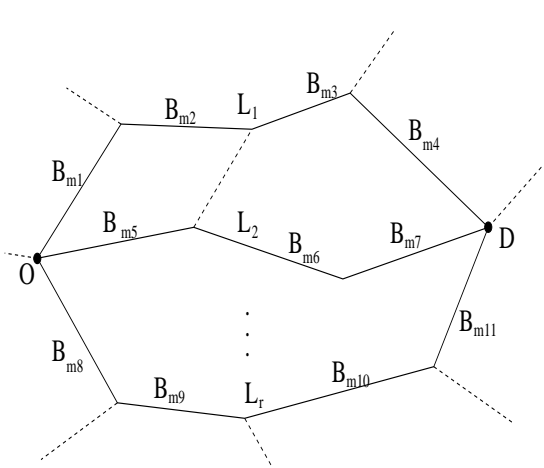

Fig. 2. Dependence of Link, Route and OD-pair Blocking Probabilities

The formulation is as follows:

The events of call-blocking on individual links used by the multicast tree are assumed to take place independently and the load offered to link $j$ is assumed to 
consists not of the total load offered but only the proportion of traffic not blocked by other links. To be more precise let $B_{m, j}$ denote the blocking probability of traffic class $m$ on link $j$, and let $E_{m, j}\left(\rho_{1}, . ., \rho_{M} ; C_{j}\right)$ be the blocking function which gives the blocking probability of traffic class $m$ on link $j$ when the offered class $m$ bandwidth demand on link $j$ is $\rho_{m}$ and link capacity is $C_{j}$. Then

$$
\begin{gathered}
B_{m j}=E_{m j}\left(\rho_{1 j}, . ., \rho_{M j} ; C_{j}\right) \text { for all } m \text { and } j \\
\lambda_{t}=\nu_{t}\left(1-L_{t}\right) \text { where }\left(1-L_{t}\right)=\prod_{m, l}\left(1-B_{m l}\right)^{A_{m l t}} \\
\rho_{m j}=d_{m j} \sum_{t}\left(1-B_{m j}\right)^{-1} \lambda_{t} A_{m j t}=d_{m j} \nu_{m, j} \text { for all } m \text { and } j
\end{gathered}
$$

where $\nu_{m, j}$ is the offered class $m$ traffic to link $j$ under the reduced load approximation. Denote that instead of routes $r$ we calculate all values for multicast trees $t$.

To obtain the point-to-multipoint connection blocking probabilities we should use the above formula but for all links the multicast tree $t$ consists of.

$$
L_{t}^{\text {exact }} \approx 1-\prod_{m, l}\left(1-B_{m l}\right)^{A_{m l t}}
$$

The link blocking probabilities $B_{m l}$ are given as a solution to (11), 12) and (13) and can be obtained by, e.g., repeated substitution.

This approach can be further generalised for multipoint-to-multipoint connections in analogous way. Furthermore, formula for $k-1$ reliable multicast can be derived as follows:

$$
L_{t}^{\text {exact }} \approx 1-\prod_{m, l}\left(1-B_{m l}\right)^{A_{m l t}}-\sum_{\forall j \supset D} B_{m j} \prod_{\forall l \in t \backslash j}\left(1-B_{m l}\right)^{A_{m l t}}
$$

where $\forall j \supset D$ stands for all those links which connect the leave nodes to the multicast tree.

By counting the number of nodes accessible via link $j$ in the multicast tree, weight $w_{j}$ can be assigned to links $j$. Then, blocking dependence for a $k$-reliable multicast can also be determined, where $k=(K-2),(K-3), \ldots, 1$ and $K$ is the number of end-points of the multicast tree.

$$
L_{t}^{\text {exact }}(k) \approx 1-\sum_{\forall \boldsymbol{p} \in\{0,1\}^{N} \text { where } \biguplus p_{q} w_{j}<K-k} \prod_{q=1}^{N} B_{m q}^{p_{q}}\left(1-B_{m q}\right)^{\overline{p_{q}}}
$$

where $\biguplus p_{q} w_{j}$ stands for the number of blocked endpoints of the multicast tree. That is, if the connection from the originator $O$ to destination node $D_{i}$ is blocked by two or more simultaneous link blockings along that path it is counted once only. $N$ is the total number of links the tree consists of. $\boldsymbol{p}=$ $\left(p_{1}, p_{2}, \ldots, p_{q}, \ldots, p_{N}\right)$ and $\overline{p_{q}}$ stands for the negation of $p_{q}$ (i.e., if $p_{q}=1 \Rightarrow$ $\overline{p_{q}}=0$, and if $p_{q}=0 \Rightarrow \overline{p_{q}}=1$ ). 


\subsection{Whitt's Summation Bound}

Formula (9) can be used to bound both, route and tree blocking probabilities for a multicast tree in way as described above for the case of reduced load approximation. The blocking probability for a node-pair connected along route $r$ can be upper bounded as follows:

$$
L_{r}^{\text {exact }} \leq \sum_{m, l} \tilde{B}_{m l} A_{m l r}
$$

where $r \subset t$, or for the case of the whole point-to-multipoint connection (i.e., multicast tree $t$ ) can be upper bounded by:

$$
L_{t}^{e x a c t} \leq \sum_{m, l} \tilde{B}_{m l} A_{m l t}
$$

\section{OD-Pair Blocking}

\subsection{The Induced Load (IL) Approximations under the Route Independence Assumption}

When Alternative Routing (AR) is applied in a network a connection setup attempt is considered blocked not after the first unsuccessful trial, but after $R$ retrials. The proposed OD-pair blocking approximations are valid for the case when the number of alternative routes $k$ of one OD-pair is equal to the number of alternative routes $|R|$ for the SAR alternative routing strategy and for any $k \leq|R|$ in case of DAR.

This approximation violates the assumption on Poissonian character of the offered traffic, because the overflow traffic becomes more peaked (bursty). For this reason the accuracy of the proposed approximation will deteriorate as the amount of the overflow traffic increases. This is valid for Alternative Routing techniques in general. However, for Dynamic Alternative Routing (DAR) the overflow is dynamically minimised, since after each unsuccessful connection establishment attempt the sequence of trials is updated, i.e., the route with high blocking probability will receive less traffic. For this reason the accuracy of the proposed approximation for DAR is excellent at moderate and low loads.

First, the approximation for Sequential AR (SAR) will be described, then that one for DAR. For the all OD-pair blocking probability approximations Route Independence is assumed, i.e., that the event of blocking a connection setup over one route does not influence the event of blocking its retrial over another route. The term Induced Load means, that the blocking of one route induces more load, i.e. the traffic will be re-offered to another route.

\subsection{OD-Pair Blocking Approximation for SAR}

The sequence of alternative routes is static. The routes are tried one-by-one in the given order until the call is established or all alternative routes exhausted. 
If all routes are unable to accommodate the connection it is considered blocked. The procedure of approximating OD-pair blocking probabilities is as follows:

1. Set the iteration counter $i=0$ and the initial route-blocking probabilities $L_{r}$ of routes $r$ to $L_{r}=0.1, \forall r \in R_{o}, \forall o \in O\left(R_{o}\right.$ is the ordered set of all routes of the OD-pair $o$; $O$ is the set of all OD-pairs within the network). Let $\nu_{o}$ be the traffic offered to OD-pair $o$ for all OD-pairs $o \in O . L_{0}^{(i)}=1$ $\forall o \in O$ in all iterations $i$.

2. $i++$;

3. Offer traffic $\nu_{o}^{(i)}(r)$ to route $r$ of the OD-pair $o$, where routes are sorted from 1 to $\left|R_{o}\right|$ according to the order of trials, where $\left|R_{o}\right|$ stands for the cardinality (number of elements) of $R_{o}$.

$$
\nu_{o}^{(i)}(r)=\nu_{o} \prod_{q=0}^{r-1} L_{q}^{(i-1)} \quad \forall r \in R_{o}, \forall o \in O
$$

4. Determine the blocking probabilities $B_{m l}$ of all links $l$ of the network for all traffic classes $m$ and that of all routes $r\left(L_{r}\right)$ for all OD-pairs $o$ iteratively using, e.g., the RL approximation as described in Sections 3 and 4 .

5. Express the OD-pair blocking probability $B_{o}$ for all OD-pairs $o$ as

$$
B_{o}^{(i)}=\prod_{\forall r \in R_{o}} L_{r}^{(i)}
$$

where $R_{o}$ is the number of both, routes and retrials for OD-pair $o$.

6. Goto Step 2 until the OD-pair blocking probabilities reach their stationary value, which is known to exist by Brouwer's fixed point theorem.

Iterative repetition of steps 2 and 3 models spreading the traffic between different routes, i.e., it ensures both, the overflow of the traffic from the first route tried to others within one OD-pair as well as the interactions between OD-pairs, since the routes of different OD-pairs share common links. The speed of convergence depends on both the average and maximal number of routes $\left|R_{o}\right|$ per OD-pair.

\subsection{OD-Pair Blocking Approximation for DAR}

The first path to be tried will always be the last path which has successfully accommodated the call. There are two alternatives for determining the sequence of alternative routes. First, the sequence is dynamically changing according the route-blocking probabilities experienced (DAR1). Second, after an unsuccessful trial a new route is randomly chosen (DAR2). The DAR1 strategy will be first investigated. Its dynamic behaviour can be modelled by the following iterative method proposed.

1. Set the iteration counter $i=0$ and the initial OD-pair blocking probability $B_{o}^{(i)}=1$. 
2. For $\forall r \in R_{o}, \forall o \in O$ set $\nu_{o}^{(0)}(r)=\frac{\nu_{o}}{\left|R_{o}\right|}$ and determine $L_{r}^{(0)}$ by, e.g., RL approximation.

3. $i++$;

4. Offer traffic $\nu_{o}^{(i)}(r)$ to the $r^{\text {th }}$ route of the OD-pair $o$ in the $i^{\text {th }}$ iteration

$$
\begin{aligned}
& \nu_{o}^{(i)}(r)=\nu_{O}\left(\frac{1-L_{r}^{(i-1)}}{\sum_{\forall z \in R_{O}, p_{z}=0}\left(1-L_{z}^{(i-1)}\right)}+\right. \\
& \left.+\sum_{\forall \boldsymbol{p} \in\{0,1\}^{\left|R_{o} \backslash r\right|}} \frac{1-L_{r}^{(i-1)}}{\sum_{\forall z \in R_{O}} p_{z}\left(1-L_{z}^{(i-1)}\right)} \prod_{\forall q \in R_{o} \backslash r} \frac{\left(1-L_{q}^{(i-1)}\right)\left(L_{q}^{(i-1)}\right)^{p_{q}}}{\sum_{\forall z \in R_{O} \backslash r}\left(1-L_{z}^{(i-1)}\right)}\right)
\end{aligned}
$$

where $\boldsymbol{p}=\left(p_{1}, p_{2}, \ldots, p_{q}, \ldots, p_{R_{o}}\right)$, and $\nu_{o}$ is the traffic originally offered to OD-pair $o$. The first term of (21) expresses that the load will be offered to route $r$ proportionally to its availability (normalised for all available paths). The second term expresses that all traffics blocked at other routes will be offered with certain probability (first factor of the second term) to route $r$. Assuming that route-blocking probabilities are small $\left(L_{q} \approx 0\right)$, the relation can be simplified:

$$
\nu_{o}^{(i)}(r)=\nu_{o} \frac{1-L_{r}^{(i-1)}}{\sum_{\forall z \in R_{o}}\left(1-L_{z}^{(i-1)}\right)}\left(1+\sum_{\forall q \in R_{o} \backslash r} \frac{\left(1-L_{q}^{(i-1)}\right) L_{q}^{(i-1)}}{\sum_{\forall z \in R_{o} \backslash r}\left(1-L_{z}^{(i-1)}\right)}\right)
$$

When determining the offered traffic to every single route $r$ (according to (22)) we implicitly assume that each unsuccessful trial is retried once only. This slightly underestimates the amount of traffic offered to routes $r$ and therefore the whole OD-pair blocking probability.

5. Determine the blocking probabilities of all links and then $L_{r}^{(i)}$ of all routes $r$ of all OD-pairs $o$ using, e.g., the RL approximation (Section 4).

6. Express the OD-pair blocking probability $B_{o}^{(i)}$ for all OD-pairs $o$ as

$$
B_{o}^{(i)}=\prod_{\forall r \in R_{o}} L_{r}^{(i)}
$$

where $R_{o}$ is the number of both, alternative routes and retrials for OD-pair $o$.

7. Go to Step 3 until blocking probabilities of OD-pairs reach their stationary value, i.e., while $\exists o \in O,\left|B_{o}^{(i-1)}-B_{o}^{(i)}\right|>\epsilon$

When this routing strategy is applied in a network the convergence of the approximation is slower than that for SAR because of iterations needed, but the accuracy is better, since the overflow traffic is minimised.

It should be mentioned, that approximation (22) is equivalent to assuming, that after an unsuccessful first attempt one retrial is allowed only, which always leads to success. According to DAR1 technique frequency of choosing a route $r$ 
among $R_{o}$ alternatives is proportional to the probability that route $r$ will not block the call, i.e., to $1-L_{r}$. Assuming that after an unsuccessful first attempt $k-1$ retrials are allowed ( $k$ trials in total), where $k<\left|R_{o}\right|$, approximation (23) can be written as:

$$
\begin{aligned}
B_{o}^{(i)}(k) & =\sum_{\forall j_{1} \in R_{o}} \frac{1-L_{j_{1}}}{\sum_{\forall l_{1} \in R_{o}}\left(1-L_{l_{1}}\right)} L_{j_{1}}\left(\sum_{\forall j_{2} \in R_{o} \backslash j_{1}} \frac{1-L_{j_{2}}}{\sum_{\forall l_{2} \in R_{o} \backslash j_{1}}\left(1-L_{l_{2}}\right)} L_{j_{2}} \ldots\right. \\
& \left.\ldots\left(\sum_{\forall j_{k} \in R_{o} \backslash\left\{j_{1}, j_{2}, \ldots, j_{(k-1)}\right\}} \frac{1-L_{j_{k}}}{\sum_{\forall l_{k} \in R_{o} \backslash\left\{j_{1}, j_{2}, \ldots, j_{(k-1)}\right\}}\left(1-L_{\left.l_{k}\right)} L_{j_{k}}\right.}\right) \ldots\right)
\end{aligned}
$$

It can be easily shown, that $B_{o}^{(i)}(k)$ is equal to (23) for $k=\left|R_{o}\right|$.

For the case of DAR2 the first trial will have the same probability as described for DAR1, but the second trial will be distributed between available (not yet tried) alternative paths with equal probabilities. For DAR2 when $k<\left|R_{o}\right|$, approximation (24) can be written as:

$$
\begin{array}{r}
B_{o}^{(i)}(k)=\sum_{\forall j_{1} \in R_{o}} \frac{1-L_{j_{1}}}{\sum_{\forall l_{1} \in R_{o}}\left(1-L_{l_{1}}\right)} L_{j_{1}}\left(\sum_{\forall j_{2} \in R_{o} \backslash j_{1}} \frac{1}{\left|R_{o}\right|-1} L_{j_{2}} \ldots\right. \\
\left.\quad \ldots\left(\sum_{\forall j_{k} \in R_{o} \backslash\left\{j_{1}, j_{2}, \ldots, j_{(k-1)}\right\}} \frac{1}{\left|R_{o}\right|-k+1} L_{j_{k}}\right) \ldots\right)
\end{array}
$$

For DAR2 it can be also easily shown, that $B_{o}^{(i)}(k)$ is equal to (23) for $k=\left|R_{o}\right|$.

\section{Conclusion and Future Work}

In this paper we have proposed new approximations for analytical evaluation of call blocking probabilities for some alternative routing techniques with different number of alternatives and retrials, as well as for full- to k-reliable multicast connections. The latter is generalised for multipoint-to-multipoint connections. The obtained results are supported by simulation results. We have shown, that the fixed routing with point-to-point connections and with no retrial is a special case of our problem, and our formula is than reduced to the reduced load approximation with link independence assumption.

The importance of these results lies in the fact that in modern telecommunication networks the number of links is much smaller than the number of routes. For network operators, link blocking values are often available and in case of reconfiguration the fast and simple route and OD pair blocking calculations can be performed. 


\section{References}

1. A. Girard, "Routing and Dimensioning of Circuit Switched Networks", AddisonWesley, 1990

2. F.P. Kelly, "Routing in Circuit-Switched Networks: Optimization, Shadow Prices and Decentralization", Advanced Applied Probability, Vol. 20, pp. 112-144, 1988

3. L. Ast, S. Blaabjerg, T. Cinkler, "Approximations for Revenue Optimization in Multi-Rate Loss Networks", ITC Seminar: New Telecommunication Services for Developing Networks, St.-Petersburg, June 1995

4. T. Cinkler, G. Fodor, L. Ast, S. Racz, "End-To-End Blocking Probability Approximations for Resource Management in Multirate Loss Networks", International Teletraffic Congress Seminar, Bangkok, 1995

5. A. Girard, "Routing and Dimensioning in Circuit Switched Networks", Addison Wesley Publishing Company, ISBN 0-201-12792-X, 1990

6. J.W. Roberts, Z. Dziong "Congestion Probabilities in a Circuit Switched Integrated Services Network", North-Holland, Performance Evaluation 7 267-284, 1987

7. K.W. Ross, "Multiservice Loss Models for Broadband Telecommunication Networks", Springer Verlag, ISBN 3-540-19918-7, 1995

8. M. Ritter, P. Tran-Gia, editors, "Multi-Rate Models for Dimensioning and Performance Evaluation of ATM Networks", Cost 242 Interim Report, June 1994

9. T. Cinkler, G. Fodor, L. Ast, S. Rácz, "End-to-End Blocking Probability Approximations for Resource Management in Multi-Rate Loss Networks", ITC Seminar, Bangkok, November 1995

10. T. Cinkler, G. Fodor, S. Rácz, T. Henk, "Simulative Analysis of End-to-End Call Blocking Probability Evaluation Techniques in Multi-Rate Loss Networks", 10th European Simulation Multiconference, Budapest, June 1996

11. L. Ast, S. Blaabjerg, T. Cinkler, G. Fodor, S. Rácz, "Blocking Probability Approximations and Revenue Optimization in Multi-Rate Loss Networks", Special Issue of "Simulation", Modelling and Simulation of Computer Systems and Networks: Part One, Vol.68, No.1, Jan 1997

12. R. Guérin, H. Ahmadi, M. Naghshineh, "Equivalent Capacity and Its Application to Bandwidth Allocation in High Speed Networks" IEEE JSAC, September 1991

13. Z. Dziong, J.W. Roberts, "Congestion Probabilities in a Circuit Switched Integrated Service Network", Performance Evaluation 7, 1987

14. Cost 242 Interim Report, M. Ritter, P. Tran-Gia (Ed.), "Multi-Rate Models for Dimensioning and Performance Evaluation of ATM Networks", Commission of the European Communities, June 1994

15. L. Delbrouck, "On the Steady-State Distribution in a Service Facility Carrying Mixtures of Traffic with Different Peakedness and Capacity Requirements", IEEE Trans. on Comm., No. 11, 1983

16. S-P. Chung, K.W. Ross, "Reduced Load Approximations for Multirate Loss Networks", ACM/IEEE Transactions on Networking, 1993, pp. 1222-1231

17. A. Faragó, S. Blaabjerg, L. Ast, T. Henk, "A New Degree of Freedom in ATM Network Dimensioning: Optimizing the Logical Configuration", IEEE JSAC, Journal on Selected Areas in Communications, 1996

18. F.P. Kelly, "Blocking Probabilities in Large Circuit-Switched Networks", Adv. Appl. Prob. Vol. 18, 1986, pp. 473-505

19. W. Whitt, "Blocking When Service Is Required From Several Facilities Simultaneously", AT\&T Technical Journal Vol. 64, No. 8, pp. 1807-1856, October 1985

20. S. Paul, Multicasting on the Internet and its Applications, 1998, Kluwer, ISBN 0-7923-8200-5 